\title{
Nutraceuticals aid in managing COVID-19
}

\author{
Sweta Sharma ${ }^{1}$, Praveen Bhai Patel ${ }^{2}$ and Arpita Yadav ${ }^{1}$ \\ ${ }^{1}$ Department of Chemistry and ${ }^{2}$ Department of Chemical Engineering, University Institute of \\ Engineering and Technology, Chhatrapati Shahu Ji Maharaj University, Kanpur 208024
}

Received: 10-01-2022 / Revised Accepted: 30-01-2022 / Published: 01-02-2022

\begin{abstract}
In this study authors have investigated some natural compounds derived from common herbs and condiments utilized for prevention and control of cough and cold symptoms. The main chemical constituents of these compounds have been docked to the main protease Mpro active site of SARS-CoV-2 to study its inhibition and hence their utility in controlling cold symptoms associated with COVID-19 infection. Molecular dynamics simulation studies have also been performed to observe the dynamical stability of best pose complexes. Ligand interactions have been analyzed and compared with co-crystallized ligand N3 interactions. Main polar residues involved are gln 189, glu 166 and thr 190. Although all natural compounds investigated show Mpro inhibition capabilities, glabridin and liquiritin which are the main constituents of licorice show best inhibitory property. Therefore, incorporation of licorice in hot beverages like tea is recommended to combat and prevent COVID-19 symptoms.
\end{abstract}

Keywords: nutraceutical, SARS CoV-2, MPro, curcumin, aloin A, cordioside, nimbin, Liquiritin, glabridin, molecular docking, MD simulations

\section{INTRODUCTION}

Scientists across the globe have come forward making all efforts in understanding, managing and combating Coronavirus disease 2019 COVID-19 in their unique ways right from design stage to market and clinical administration. Apart from drugs and vaccines we have forever looked upon nutraceuticals as an easy source of age old medications and preventive measures. COVID-19 patients show symptoms similar to viral fever and pneumonia [1,2]. Therefore, it is only obvious to consider natural compounds used in pneumonia and viral fever. Among the popular choices are aloe vera, basil, neem, licorice, curcumin and giloy [38]. We have selected some of the main constituents of these natural compounds and studied their possible inhibitory mode of action on the replication of coronavirus. The coronavirus implicated in COVID-19 is termed as Severe acute respiratory syndrome SARS CoV-2 virus. Its genome houses several proteins involved with its infection and replication process such as spike, main protease Mpro and RNA-dependent RNA

Address for Correspondence: Arpita Yadav, Department of Chemical Engineering, University Institute of Engineering and Technology, Chhatrapati Shahu Ji Maharaj University, Kanpur 208024, India; E-mail: arpitayadav@yahoo.co.in

How to Cite this Article: Sweta Sharma, Praveen Bhai Patel and Arpita Yadav. Nutraceuticals aid in managing COVID-19. World J Pharm Sci 2022; 10(02): 183-190;

https://doi.org/10.54037/WJPS.2022.100204

Copyright:2022@ The Author(s). This is an open access article distributed under the terms of the Creative Commons AttributionNonCommercial-ShareAlike 4.0 International License (CC BY-NC-SA), which allows re-users to distribute, remix, adapt, and build upon the material in any medium or format for noncommercial purposes only, and only so long as attribution is given to the creator. If you remix, adapt, or build upon the material, you must license the modified material under identical terms. 
polymerase [9]. Mpro facilitates the cleavage of viral peptides into smaller functional units involved in replication process [10]. Since coronaviruses were also implicated in Middle east respiratory syndrome (MERS), inhibitors of Mpro are not new. A comprehensive study by Liu et al summarizes Mpro inhibitors [11]. The study summarizes all peptidic and non-peptidic small molecule inhibitors of Mpro. At the same time it also discusses different natural compounds explored for inhibition of Mpro till 2020. It gives a summary of studies performed on flavonoids, chalcones, terpenoid derivatives and isatin derivatives. Here we investigate Mpro inhibitory properties of main constituents of some natural compounds specifically useful in viral fever and infections as mentioned above.

Turmeric is well known for its healing properties which are largely due to its constituent curcumin [12]. One of the main active constituents of aloe vera is a anthraquinone compound Aloin A [13], that of giloy is a terpenoid cordioside [14]. Main constituent of neem is nimbin [15]. The antimicrobial activity of licorice have been mainly attributed to phenolic and flavonoid compounds present such as liquiritin and glabridin [16]. The chemical structures of these constituents are shown in fig. 1.

\section{METHODOLOGY}

The selected natural compounds were prepared using the protein preparation wizard of Maestro interface. The X-ray coordinates for the target that is the main protease of SARS-CoV-2 Mpro were taken from pdb file 6LU7 at $2.16 \AA$ resolution without any missing residues [17]. The protein was prepared by adding polar hydrogens using Auto Dock version 4 [18] and Auto Dock Vina Version 1.1.2 [19]. The co-crystallized peptidomimetic ligand N3 was re-docked with RMSD less than 2.0 $\AA$ to authenticate grid placement for dockings. The prepared natural compounds or the ligands were then docked one by one in the active site of Mpro and their interactions with the active site residues were analysed in detail. Auto Dock Vina was used to perform flexible ligand docking studies. Best poses obtained were exported to Shaw's Desmond software [20] to perform simulation studies. Standard molecular dynamics protocol was followed on best binding energy complexes to study the dynamical behaviour and stability of complex with time. All atom SPC water was incorporated in a grid of $10 \times 10 \times 10 \AA$ around the protein and orthorhombic boundary conditions were applied. The minimized complex were subjected to simulated annealing followed by dynamics at $300 \mathrm{~K}$ for $25 \mathrm{~ns}$. NPT ensemble was chosen utilizing Martyna-Tobias-Klein barotat method [21]. The RESPA integrator was used with $2 \mathrm{fs}$ time step for near and bonded and $6 \mathrm{fs}$ time step for far atoms [22]. Coulombic interaction cutoff was set at $9 \AA$. RMSD analysis has been performed for all heavy atoms together to judge the stability of the system overtime and fluctuations in position of ligand. Deviations have been taken with respect to initial frame for dynamics.

\section{RESULTS AND DISCUSSION}

The chemical structures of studied natural compounds are shown in fig. 1. These compounds were prepared as mentioned in the methods section. The choice of these natural compounds is based on the age old ayurvedic Indian tradition of utilizing these natural compounds to cure common cough and cold symptoms. The main protease protein of SARS-CoV-2 Mpro was also prepared using ADT tools associated with Auto Dock software. The center of grid for docking studies was placed at cocrystallized ligand N3 and authenticated by redocking N3 with a low RMSD as mentioned above. The affinity of N3 is given in table 1 and interactions of re-docked N3 with Mpro active site residues are shown in fig. 2 and listed in table 2 . A $2 \mathrm{D}$ interaction map is given in fig. 3. After grid authentication prepared compounds were docked one by one. Top nine poses with best docking scores were filtered for each compound. Table 1 lists the best affinities obtained for each compound. As can be seen at least the top three compounds derived from licorice and neem show affinities quite close to the co-crystallized peptidomimetic ligand N3. The detailed interactions of the best affinity compound glabridin are shown in fig. 4 . The main electrostatic interactions involved are with gln189, asn 142 and glu 166. There is efficient hydrogen bonding with thr 190, ser 144 and hid 163. Apart from these important hydrophobic interactions are also observed with pro 168 and phe 140. These interactions together hold glabridin in place so as to block the Mpro active site. Similarly, the interactions of other docked natural compounds with good docking score were analyzed. The 2D ligand interaction maps of top 6 docking score compounds are shown in figs. 5 and 6 . The main interacting residues are listed out in table 2 . Although all compounds dock at the same place in different orientations utilizing the same polar residues (gln 189, glu 166, thr 190 in particular); the ones with lower docking score do not interact very efficiently due to their orientation. The molecular size of glabridin and conformation are most appropriate for MPro inhibition. Although Aloin A, curcumin are lined by many more interacting polar and hydrophobic residues; the 


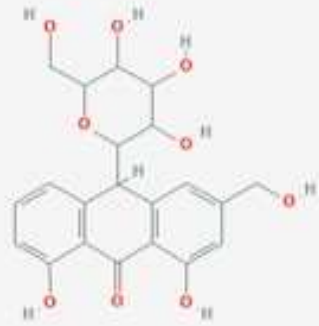

Aloin A

Source: aloe vera

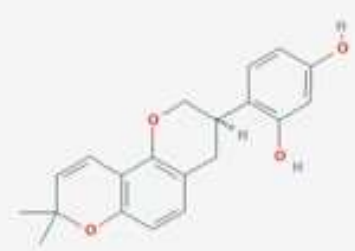

Glabridin

Source: licorice

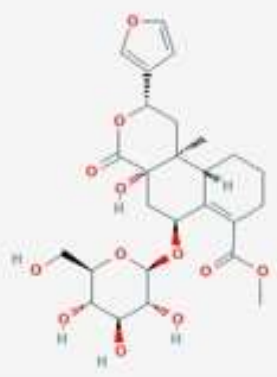

Cordioside Source: gilov

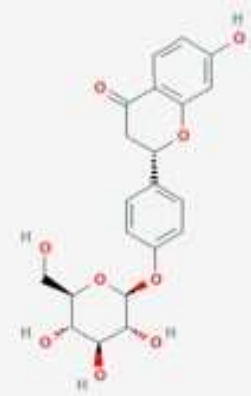

Liquiritin Source: licorice

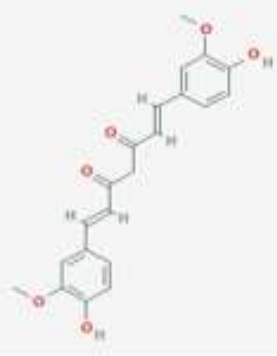

Curcumin Source: turmeric

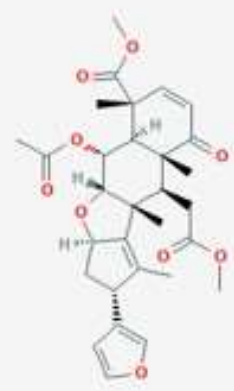

Nimbin Source: neem

Fig. 1 Chemical structures of some natural compounds useful in cough, cold and fever

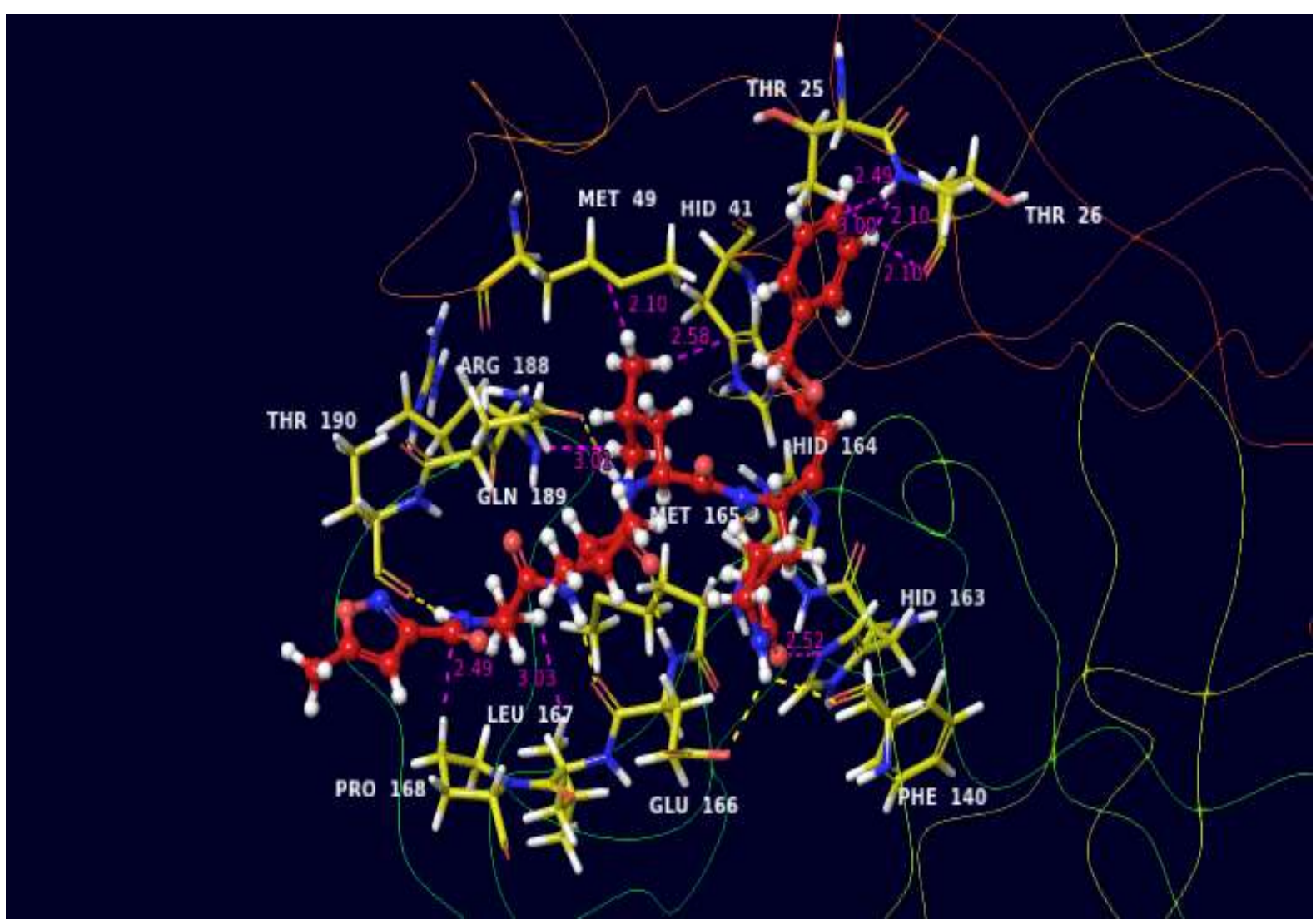


Arpita et al., World J Pharm Sci 2022; 10(02): 183-190

Fig. 2 Interactions of N3 ligand with Mpro active site residues

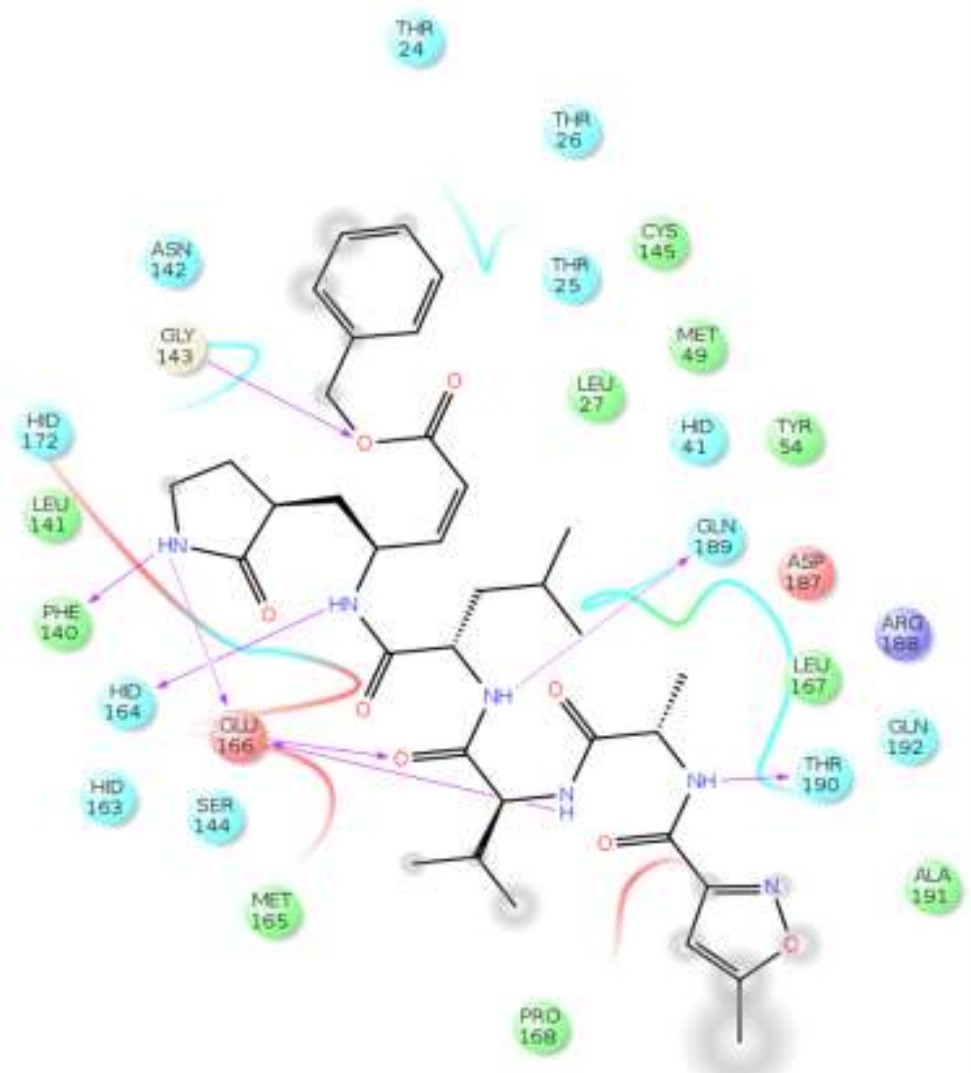

Fig. 3 2D interaction map for N3 with Mpro active site residues

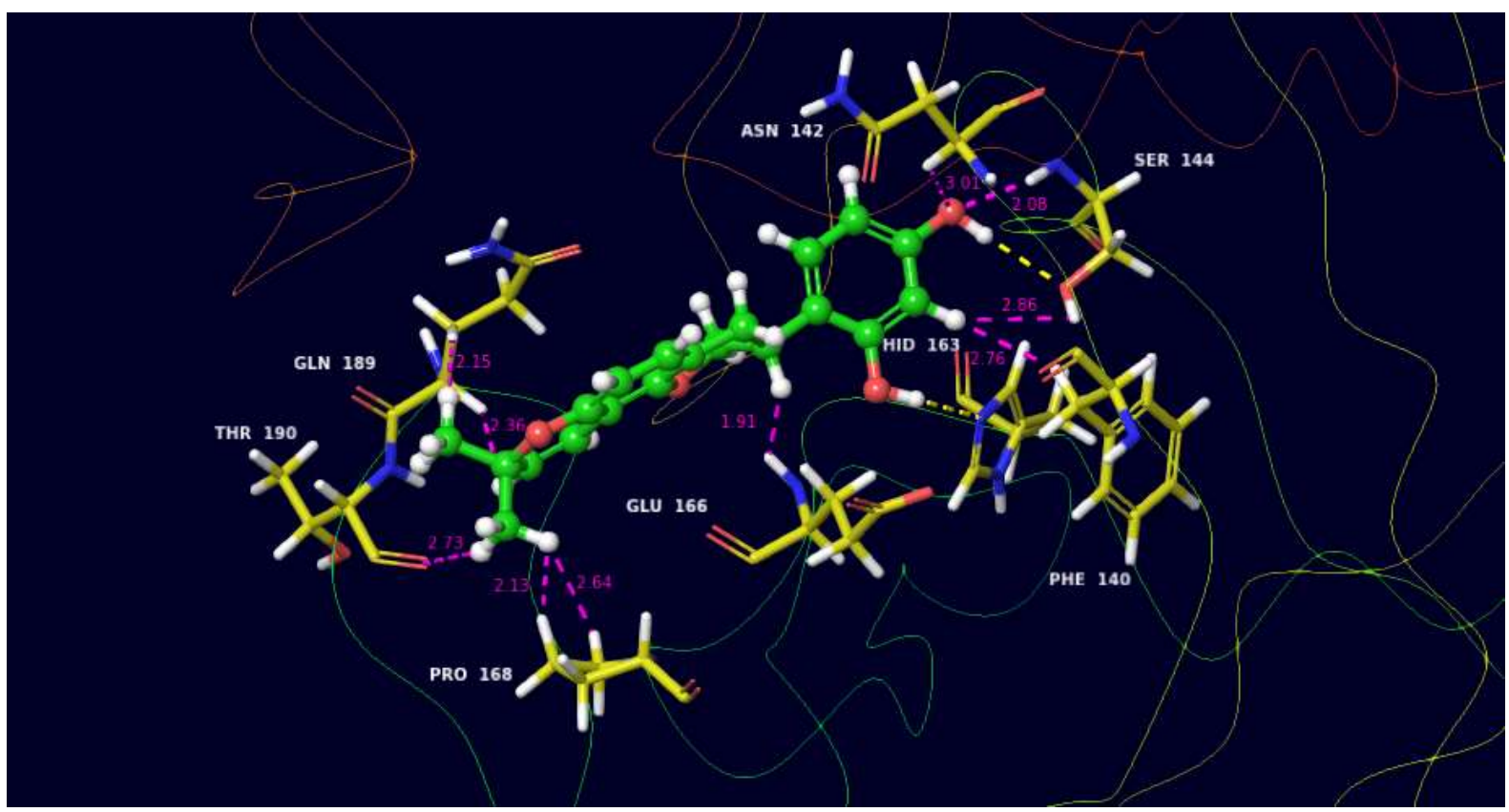

Fig. 4 Interactions of glabridin with Mpro active site residues 


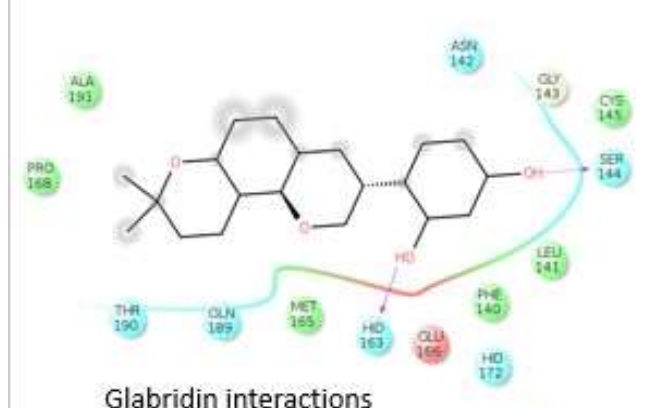

Glabridin interactions

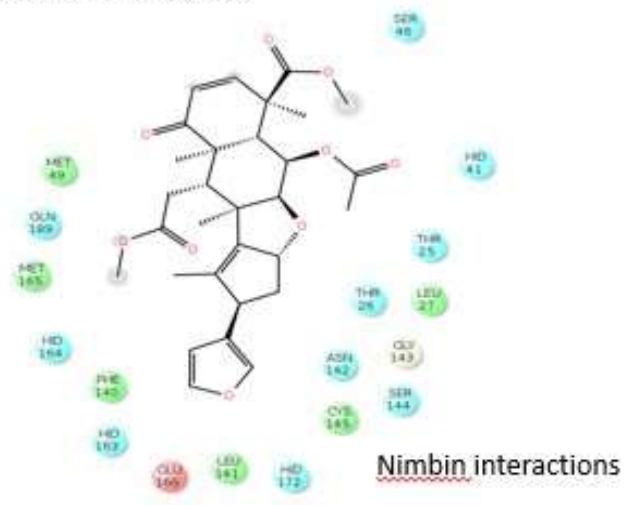

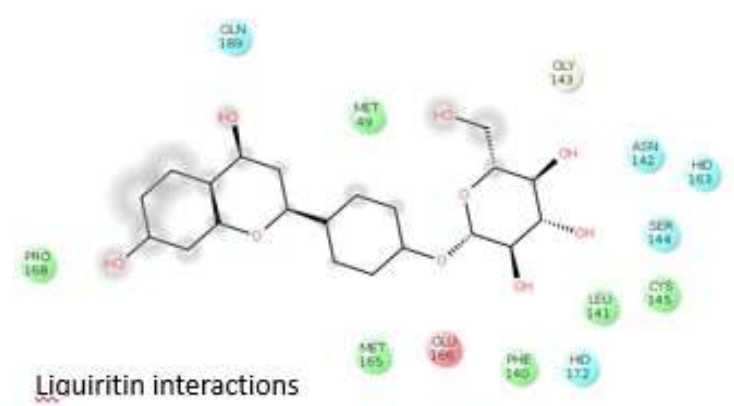

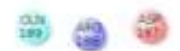

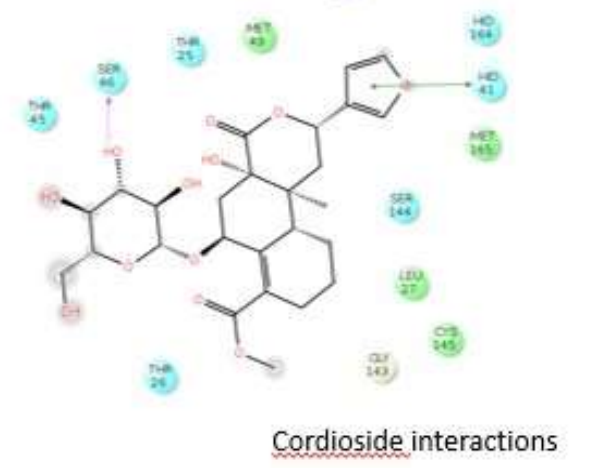

Fig. 5 Ligand interactions with Mpro active site residues

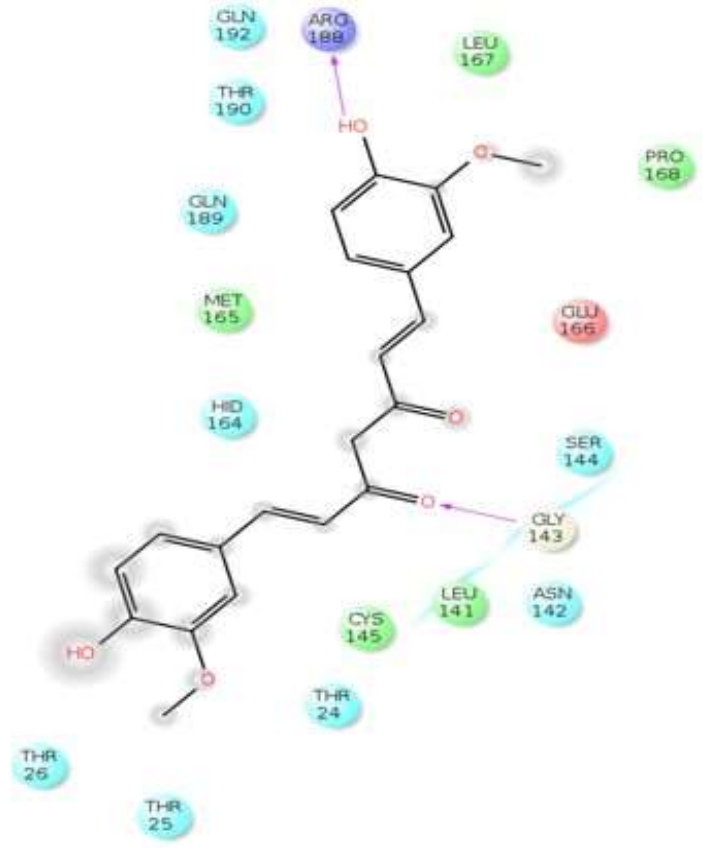

Curcumin interactions

Fig. 6 Ligand interactions with Mpro active site residues

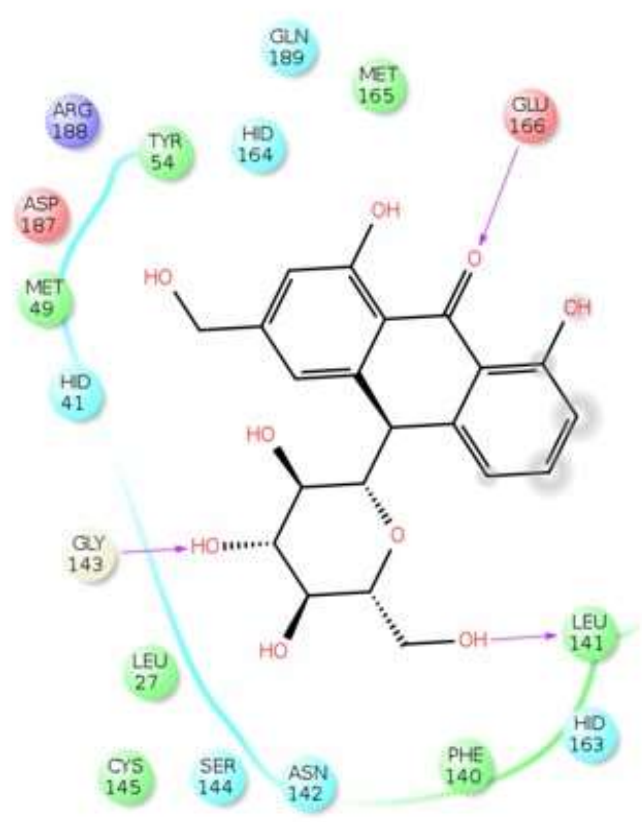

$H 1 D$
172

Aloin A interactions 
Table 1. Docking results for some natural compounds in Mpro

\begin{tabular}{|l|l|}
\hline Compound name & Affinity $(\mathrm{kCal} / \mathrm{mol})$ \\
\hline Redocked N3 & -13.6 \\
Glabridin & -9.0 \\
Liquiritin & -9.0 \\
Nimbin & -8.7 \\
Cordioside & -8.5 \\
Curcumin & -8.4 \\
Aloin A & -8.1 \\
Berberine & -7.4 \\
Gingerol & -7.1 \\
Pipernonaline & -6.6 \\
\hline Linalool & -4.8 \\
\hline Nimbin & -8.7 \\
\hline Aloin A & -8.1 \\
\hline Cordioside & -8.5 \\
\hline
\end{tabular}

Table 2. Detailed contact analysis for best pose of different ligands

Ligand

N3

Glabridin

Liquiritin

Nimbin

Cordioside

Curcumin

Aloin A
Interacting residues in Mpro active site

Gln189, Thr190, Glu166, Hid164, Phe140, Gly143, Asp187, Arg188

Gln189, Thr190, Glu166, Hid163, Ser144, Gly143

Gln189, Glu166, Hid163, Ser144, Gly143

Gln189, Glu166, Hid163, Hid164, Phe140, Gly143, Ser144, Hid41

Gln189, Asp 187, Arg188, Hid164, Gly143, Ser144, Hid41, Ser46, Thr26

Arg188, Gln189, Thr190, Glu166, Hid164, Gly143, Ser144, Pro168, thr26

Glu166, Asp187, Arg188, Leu141, Gly143, Phe140, Hid163, Asn142, Hid41, Met49, Tyr54
Docking studies can be deceptive due to their nondynamical nature and hence lack of stability check over time. We have therefore performed molecular dynamics simulation studies on best docked poses for observing stability of interactions with active site residues over time for the six filtered compounds. $25 \mathrm{~ns}$ trajectories have been generated with the exceptions of best and a moderately interacting compound for which longer trajectories of $50 \mathrm{~ns}$ have been observed as per the protocol mentioned in methods section. The overall RMSD for all heavy atoms with respect to initial frame are shown in figs. 7 and 8. In general the overall RMSD for all heavy atoms is below $3 \AA$ which indicates low fluctuations in position of ligands and good stability of complexes over time. Molecular dynamics simulation results show that studied natural compounds possess the capability to inhibit Mpro enzyme of SARS-CoV-2 thus retarding the replication of coronavirus responsible for COVID19 infection. Although all studied compounds show inhibitory property, the phenolic flavonoids glabridin and liquiritin derived from licorice seem to be the best nutraceuticals based on molecular level study in combating and preventing COVID19 infections. Licorice which is a famous constituent of lozenges consumed during throat irritation and infection is recommended to be incorporated in everyday tea preparations as a preventive measure for common cough and cold infections as well as COVID-19 infections.

\section{Concluding Remarks}

In this study we have shown the inhibitory activity of some natural compounds derived from commonly used herbs and spices against cough and cold symptoms induced by SARS-CoV-2 infection. Though all the studied compounds showed activity against main protease enzyme Mpro of SARSCoV-2 virus involved in its replication; two phenolic flavonoid compounds glabridin and liquiritin which are main constituents of licorice have been recommended as food supplements for protection against COVID-19 infections. This recommendation is based on molecular level docking studies and molecular dynamics simulations on best pose complexes to study their stability in active site over time.

\section{Acknowledgements}

The authors gratefully acknowledge financial support from Science and Engineering Research Board (SERB), New Delhi (Project no. EMR/2016/000769). Ms. Sweta Sharma also thanks SERB for Senior Research Fellowship. Infrastructural support from University Institute of Engineering and Technology, Chhatrapati Shahu Ji Maharaj University, Kanpur is gratefully 
acknowledged. We also gratefully acknowledge ongoing collaborative research on COVID-19 with

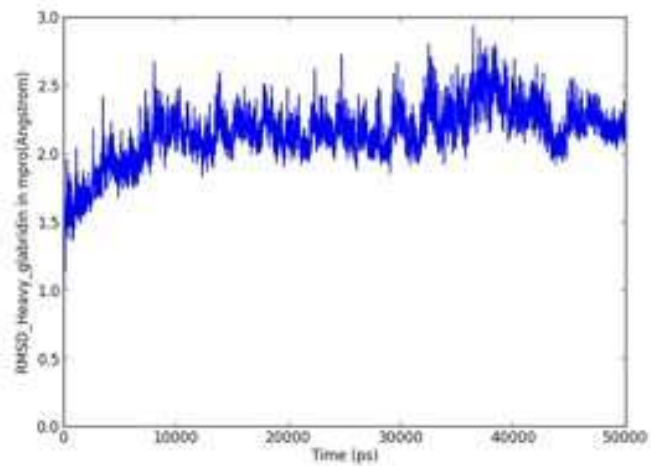

a. Glabridin

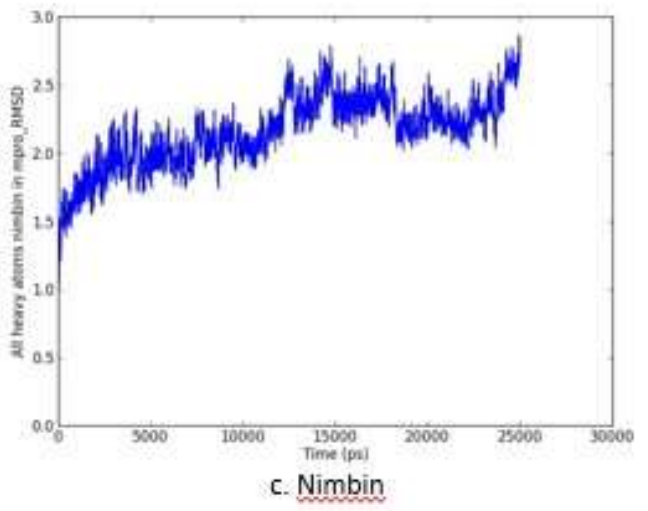

Prof. Poirier's lab at Memorial University of Newfoundland, St. John's, Canada.

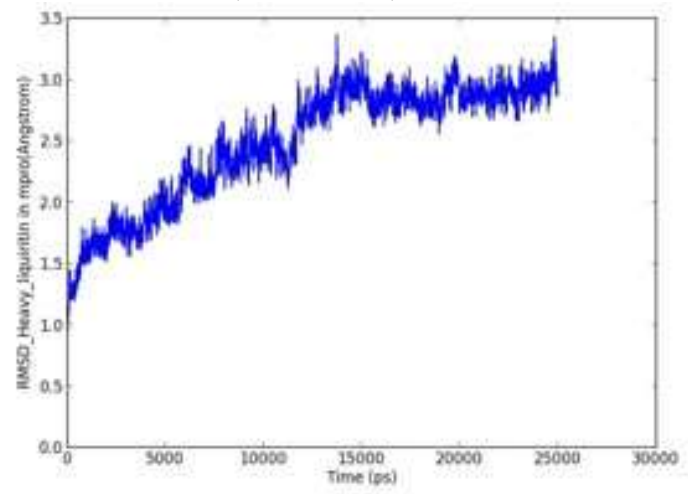

b. Liquiritin

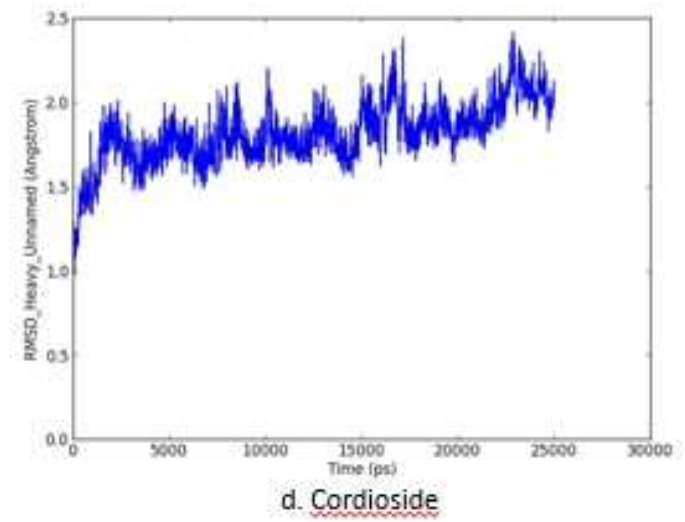

Fig. 7 Root mean square deviation for all heavy atoms with respect to initial frame for best pose complex of different natural compounds in Mpro active site

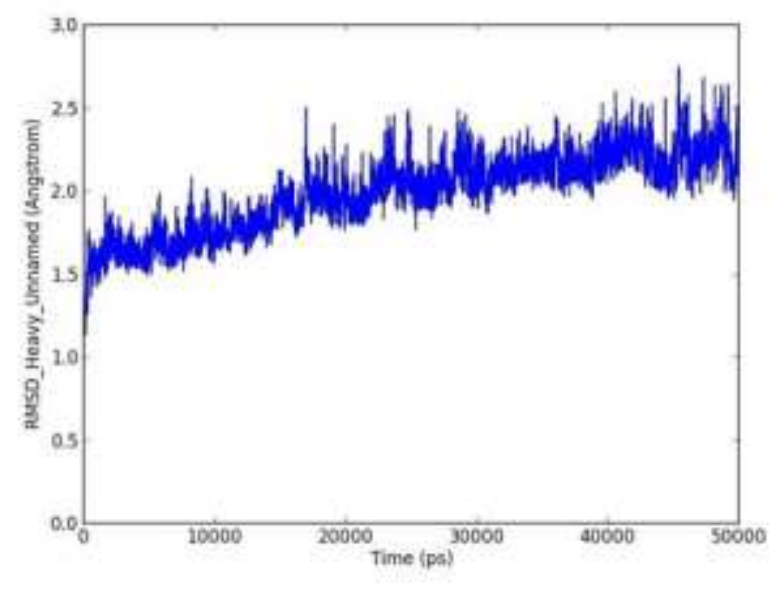

e. Curcumin

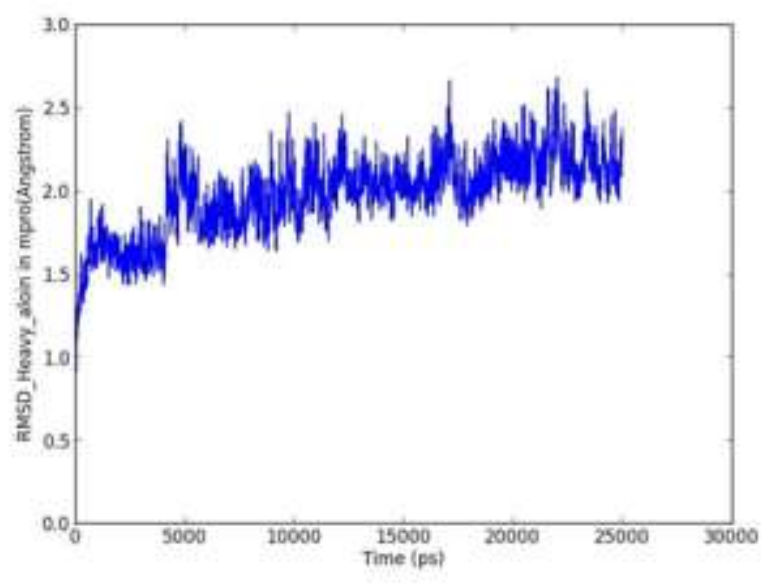

f. Aloin A

Fig. 8 Root mean square deviation for all heavy atoms with respect to initial frame for best pose complex of different natural compounds in Mpro active site

Conflict of Interests: The authors declare no competing conflicts of interest

\section{References}

1. Guan, W.J. et al. Clinical characteristics of coronavirus disease 2019 in China. N. Engl. J. Med., 2020; 382: $1708-1720$. 
2. Huang, C. et al. Clinical features of patients infected with 2019 novel coronavirus in Wuhan, China. Lancet, 2020; 395: 497-506.

3. Safa, O. et al. Effects of licorice on clinical symptoms and laboratory signs in moderately ill patients with pneumonia from COVID-19: A structured summary of a study protocol for a randomized controlled trial. Trials, 2020; 21: 790.

4. Chiang, L.C. et al. Antiviral activities of extracts and selected pure constituents of Ocimum basilicum. Clin. Expt. Pharmacol. Physiol., 2005; 32(10): 811-816.

5. Rao, V.B.; Yeturu, K. bioRxiv, doi: https://doi.org/10.1101/2020.04.29.069567, April 2020.

6. Yagi, A.; Hasegawa, M.; Ataka, S. Beneficial efficacy of aloe vera to viral infections: Case reports of Kampo medicine with aloe vera juice. J. Gastroenterol. Hepato. Res., 2020; 9(4): 3242-3247.

7. Mathew, D.; Hsu, W.L. Antiviral potential of curcumin. J. Funct. Foods, 2018; 40: 692-699.

8. Pruthvish, R.; Gopinatha, S.M. Antiviral prospective of Tinospora cordifolia on HSV-1. Int. J. Curr. Microbiol. App. Sci., 2018; 7(1): 3617-3624.

9. Liu, C. et al. Research and development on therapeutic agents and vaccines for COVID-19 and related human coronavirus diseases. ACS Cent. Sci., 2020; 6(3): 315-331.

10. Goetz, D.H. et al. Substrate specificity profiling and identification of a new class of inhibitor for the major protease of the SARS coronavirus. Biochemistry, 2007; 46: 8744-8752.

11. Liu, Y. et al. The development of coronavirus 3C-like protease (3CL pro) inhibitors from 2010 to 2020. Eur. J. Med. Chem., 2020; 206: 112711-112713.

12. Dosoky, N.S.; Setzer, W.N. Chemical composition and biological activities of essential oils of Curcuma species. Nutrients, 2018; 10: 1196.

13. Kar, S.K.; Bera, T.K. Phytochemical constituents of Aloe Vera and their multifunctional properties: A comprehensive review. Intl. J. Pharm. Sci. Res. (IJPSR), 2018; 9(4): 1416-1423.

14. Sharma, P. Et al. The chemical constituents and diverse pharmacological importance of Tinospora cordifolia. Heliyon, 2019; 5: e02437.

15. Sarah, R. et al. Natural Bio-active compounds Volume 1: Production and Applications, Eds: Akhtar, M.S.; Swamy, M.K.; Sinnaly, U.R. 2019; Chapter 17: pg 509, Springer Nature Singapore Pte Ltd..

16. Mamedov, N.A.; Egamberdieva, D. in Plant and human health, Eds: Ozturk, M.; Hakeem, K.R. 2019; Chapter 1: vol. 3, 1-21, Springer Nature Switzerland AG.

17. Jin, Z. et al. Structure of $\mathrm{M}^{\text {pro }}$ from SARS-CoV-2 and discovery of its inhibitors. Nature, 2020; 582: 289-293.

18. Morris, G.M. et al. Automated docking using a Lamarckian genetic algorithm and an empirical binding free energy function.J. Comput. Chem., 1998; 19(14): 1639-1662.

19. Trott, O.; Olson, A.J. AutoDock Vina: Improving the speed and accuracy of docking with a new scoring function, efficient optimization, and multithreading. J. Comput. Chem., 2010; 31(2): 455-461.

20. Bowers, K.J. et al. Scalable algorithms for molecular dynamics simulations on commodity clusters. Proceedings of the ACM/IEEE conference on supercomputing (SC06) ACM, Tampa, Florida, 2006; Nov 11-17.

21. Martyna, G.J.; Tobias, D.J.; Klein, M.L. Constant pressure molecular dynamics algorithms. J. Chem. Phys. 1994; 101: 4177-4189.

22. Tuckerman, M.; Berne, B.J.; Martyna, G.J. Reversible multiple time scale molecular dynamics. J. Chem. Phys. 1992; 97(3): 1990-2001. 